\title{
Simple 1-mm receivers with fixed tuned double sideband SIS mixer and wideband InP MMIC amplifier
}

Gregory A. Engargiola, Alessandro Navarrini, Richard L. Plambeck, N. Wadefalk

Gregory A. Engargiola, Alessandro Navarrini, Richard L. Plambeck, N. Wadefalk, "Simple 1-mm receivers with fixed tuned double sideband SIS mixer and wideband InP MMIC amplifier," Proc. SPIE 5498, Millimeter and Submillimeter Detectors for Astronomy II, (8 October 2004); doi: $10.1117 / 12.552401$

Event: SPIE Astronomical Telescopes + Instrumentation, 2004, Glasgow, United Kingdom 


\title{
Simple $1 \mathrm{~mm}$ receivers with fixed tuned double sideband SIS mixer and wideband InP MMIC amplifier
}

\author{
G. Engargiola ${ }^{\mathrm{a}}$, A. Navarrini ${ }^{\mathrm{a}}$, R. L. Plambeck ${ }^{\mathrm{a}}$, N. Wadefalk ${ }^{\mathrm{b}}$ \\ ${ }^{a}$ Radio Astronomy Laboratory, University of California, Berkeley, CA 94720 \\ ${ }^{b}$ Electrical Engineering Department, California Institute of Technology, Pasadena CA 91125
}

\begin{abstract}
We report on attempts to broaden the IF bandwidth of the BIMA $1 \mathrm{~mm}$ SIS receivers by cascading fixed tuned doublesideband (DSB) SIS mixers and wideband MMIC IF amplifiers. To obtain the flattest receiver gain across the IF band we tested three schemes for keeping the mixer and amplifier as electrically close as possible. In Receiver I, we connected separate mixer and MMIC modules by a 1" stainless steel SMA elbow. In Receiver II, we integrated mixer and MMIC into a modified BIMA mixer module. In Receiver III, we devised a thermally split block where mixer and MMIC can be maintained at different temperatures in the same module. The best average receiver noise we achieved by combining SIS mixer and MMIC amplifier is $45-50 \mathrm{~K}$ DSB for $v_{\mathrm{LO}}=215-240 \mathrm{GHz}$ and below $80 \mathrm{~K} \mathrm{DSB}$ for $v_{\mathrm{LO}}=205-270 \mathrm{GHz}$. The receiver noise can be made reasonably flat over the CARMA IF band $\left(v_{\mathrm{IF}}=1-5 \mathrm{GHz}\right)$. Noise temperatures for all three receivers are comparable to or better than those obtained with the BIMA receiver .
\end{abstract}

\section{INTRODUCTION}

The Berkeley-Illinois-Maryland Array (BIMA) and the Caltech Owens Valley Radio Observatory Array telescopes will merge to form the Combined Array for Research in Millimeter-Wave Astronomy (CARMA) in 2005. Each element of the new telescope ${ }^{1}$ will be equipped with a cooled $1 \mathrm{~mm}$ receiver consisting of a Superconductor-Insulator-Superconductor (SIS) mixer with Intermediate Frequency (IF) postamplifier. Downconverted signals from each element will be processed by a correlator with a total bandwidth of $4 \mathrm{GHz}$. Whereas the current OVRO $1 \mathrm{~mm}$ receivers ${ }^{2}$ cover a $4 \mathrm{GHz}$ IF band, from $1-5 \mathrm{GHz}$, the present BIMA receivers use IF postamplifiers which cover only $1.4-2.2 \mathrm{GHz}$ (L band). Before integrating BIMA instrumentation with CARMA, receiver IF bandwidths must be expanded to at least the CARMA correlator bandwidth. The resulting wide instrumental bandwidth will greatly facilitate the study of molecular transitions and continuum radiation. This paper describes our progress towards building wideband receivers by combining existing 1 mm SIS mixer chips developed for BIMA with $0.5-11 \mathrm{GHz}$ MMIC amplifiers developed for the Allen Telescope Array.

\section{NARROW IF BAND 1 MM RECEIVER AT BIMA}

The $1 \mathrm{~mm}$ SIS mixer design for BIMA was adapted by G. Engargiola and R.L. Plambeck ${ }^{3}$ from a mixer design developed by Blundell et al. ${ }^{4}$ for the Submillimeter Array. G. Engargiola fabricated the SIS chips at the Material Research Laboratory at the University of Illinois, Urbana-Champaign. Figure 1 shows a photo of the SIS chip in an open mixer block with circuit elements labeled. A series $\sim \lambda / 8$ superconducting microstrip stub $\mathrm{RF}$ tunes the $\mathrm{Nb} / \mathrm{Al}^{-}-\mathrm{Al}_{2} \mathrm{O}_{3} / \mathrm{Nb} 1.2 \times 1.2$ $\mu \mathrm{m}^{2}$ SIS junction. A butterfly probe in a half height waveguide feeds RF/LO signals to the junction through a two-section microstrip quarter-wave transformer. The waveguide cavity behind the antenna probe is a fixed backshort which greatly simplifies the mixer operation. Representative I-V characteristics, shown next to the photo, indicate a range of device characteristics resulting from junction and circuit dimension variations. Despite this range, all $1 \mathrm{~mm}$ receivers at BIMA have remarkably similar noise temperatures.Very often, differences can be traced to slight misalignment of the waveguide probe relative to the backshort. Empirically, we found that receiver noise temperature depends only weakly on the real impedance match between the mixer and $50 \Omega$ coaxial line linking the L band LNA so we used a short section of $50 \Omega$ microstrip on Duroid 5880 to couple the mixer IF pad to its SMA output connector.

Figure 2 shows receiver temperature versus frequency for a typical BIMA receiver. Best sensitivities occur between 215 and $240 \mathrm{GHz}$ where $\mathrm{T}_{\text {rec,DSB, DSB }}<55 \mathrm{~K}$. Mixer operating temperatures are usually $3.6-4.5 \mathrm{~K}$; over this range, $\mathrm{T}_{\text {rec,DSB }}$ varies less than $5 \mathrm{~K}$ Reasonable mixer performance occurs for $v_{\mathrm{LO}} \sim 205-270 \mathrm{GHz}$, where $\mathrm{T}_{\text {rec,DSB }}<80 \mathrm{~K}$. 
At least two considerations led us to surmise that the BIMA mixers could be modified to give a wider IF bandwidth. An approximation of the quality parameter $\mathrm{Q}_{\mathrm{IF}} \cong \omega \mathrm{Z}_{\mathrm{IF}}\left(\mathrm{C}_{\mathrm{j}}+\mathrm{C}_{\text {match }}\right) \sim 1.2$ at $\mathrm{GHz}$ can be made from estimates of the pumped impedance of the junction $\left(\sim 10 * \mathrm{R}_{\mathrm{n}} \cong 180 \Omega\right)$ in parallel with the parasitic junction capacitance $\left(\mathrm{C}_{\mathrm{j}} \sim 115 \mathrm{fF}\right)$ and the low frequency capacitance of the RF matching network $(\sim 250 \mathrm{fF})$. The low value suggests that a wide relative IF band can be
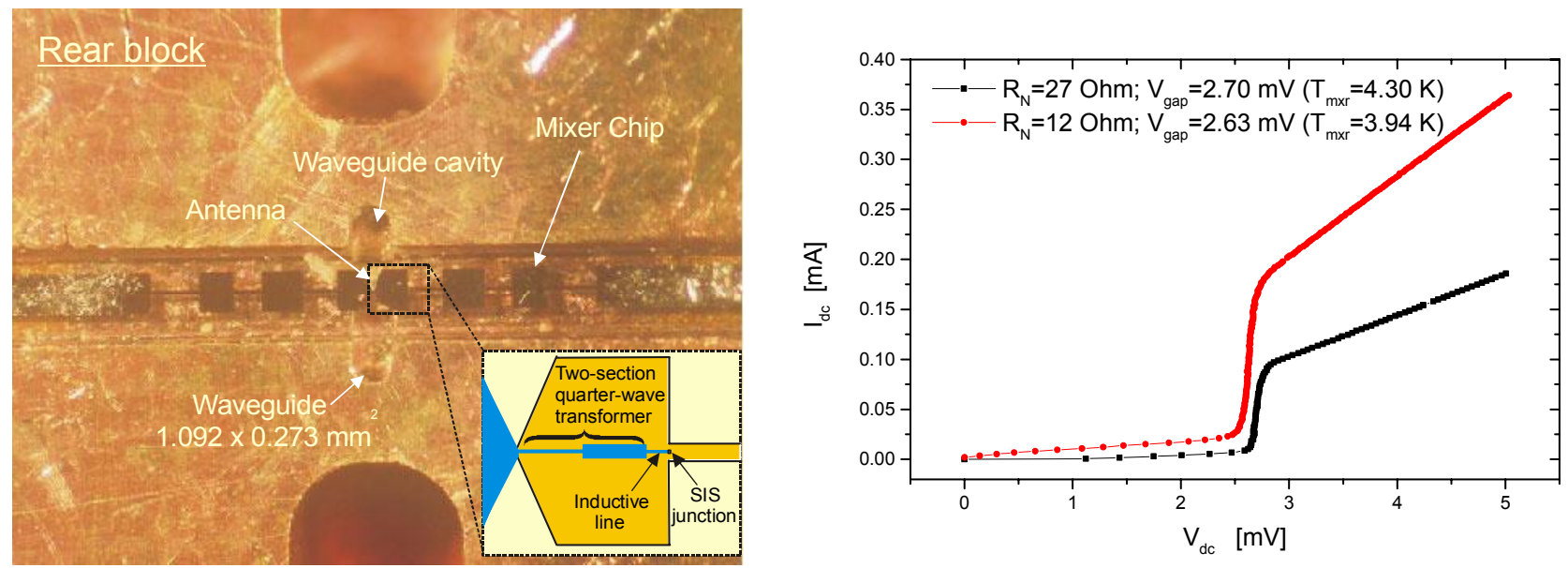

Figure 1 (left) Close-up view of the BIMA SIS mixer chip and (right) typical I-V characteristics for these devices.

covered using this mixer within the specifications of the CARMA project. Secondly, circuit elements of the BIMA mixer were adapted from the SMA $1 \mathrm{~mm}$ design, which operates with a similar IF bandwidth but centered at $5 \mathrm{GHz}$. Hence we speculated that an IF network could be devised so that the mixer would work well from the low frequency edge of the BIMA IF band $(1.4 \mathrm{GHz})$ to the high frequency edge of the SMA IF band $(6 \mathrm{GHz})$. Widening the IF band of our SIS $1 \mathrm{~mm}$ receiver without degrading its instantaneous band noise performance can most simply be attempted by connecting the mixer to a wideband MMIC amplifier with suitable bandwidth, high gain, and low noise.

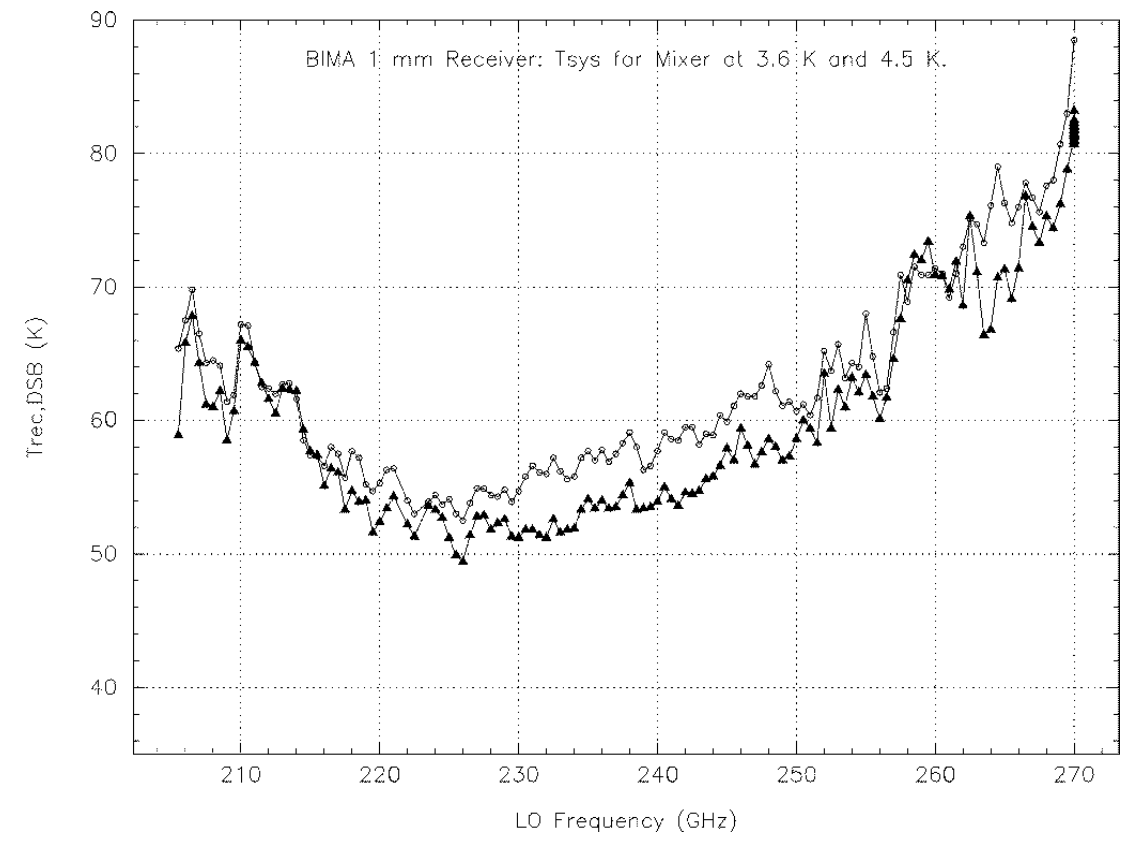

Figure 2 Receiver temperature versus LO frequency for BIMA $1 \mathrm{~mm}$ receiver with L band IF amplifier. Filled triangles denote mixer operating at $3.6 \mathrm{~K}$; open circles denote mixer operating at $4.5 \mathrm{~K}$. 


\section{WIDEBAND MMIC AMPLIFIERS}

Kerr et al. ${ }^{5}$ and Lauria et al. ${ }^{6}$ have produced $1 \mathrm{~mm}$ receivers with $4-12 \mathrm{GHz}$ IF by following SIS mixers with amplifiers built from discrete transistors. We hope to use MMIC IF amplifiers instead. Wadefalk and Weinreb developed two MMIC (WBA12 and WBA13) devices for the Allen Telescope Array ${ }^{7}$ which should be well-suited for CARMA IF postamplifiers The WBA12 and WBA13 include either two or three $0.1 \mu \mathrm{m}$ InP HEMT stages tuned to be unconditionally stable when attached to any passive load. The chip size is $2 \times 0.7 \sim \mathrm{mm}^{2}$. At a $10 \mathrm{~K}$ operating temperature the WBA12 and 13 have a noise temperature of $\mathrm{T}_{\mathrm{n}} \sim 4 \mathrm{~K}$ and gain of $\sim 25 \mathrm{~dB}$ and $\sim 35 \mathrm{~dB}$, respectively, across a $3-11$ $\mathrm{GHz}$ band. At $1 \mathrm{GHz}, \mathrm{T}_{\mathrm{n}} \sim 8 \mathrm{~K}$ and gain is $5 \mathrm{~dB}$ lower for both. Figure 3 shows a WBA13 chip, with RF input attached to a $50 \Omega$ matching network and the output attached to a $50 \Omega$ microstrip. The gates are biased through 10:1 voltage dividers and the drain wire provides $10-20 \mathrm{~mW}$ of DC power.
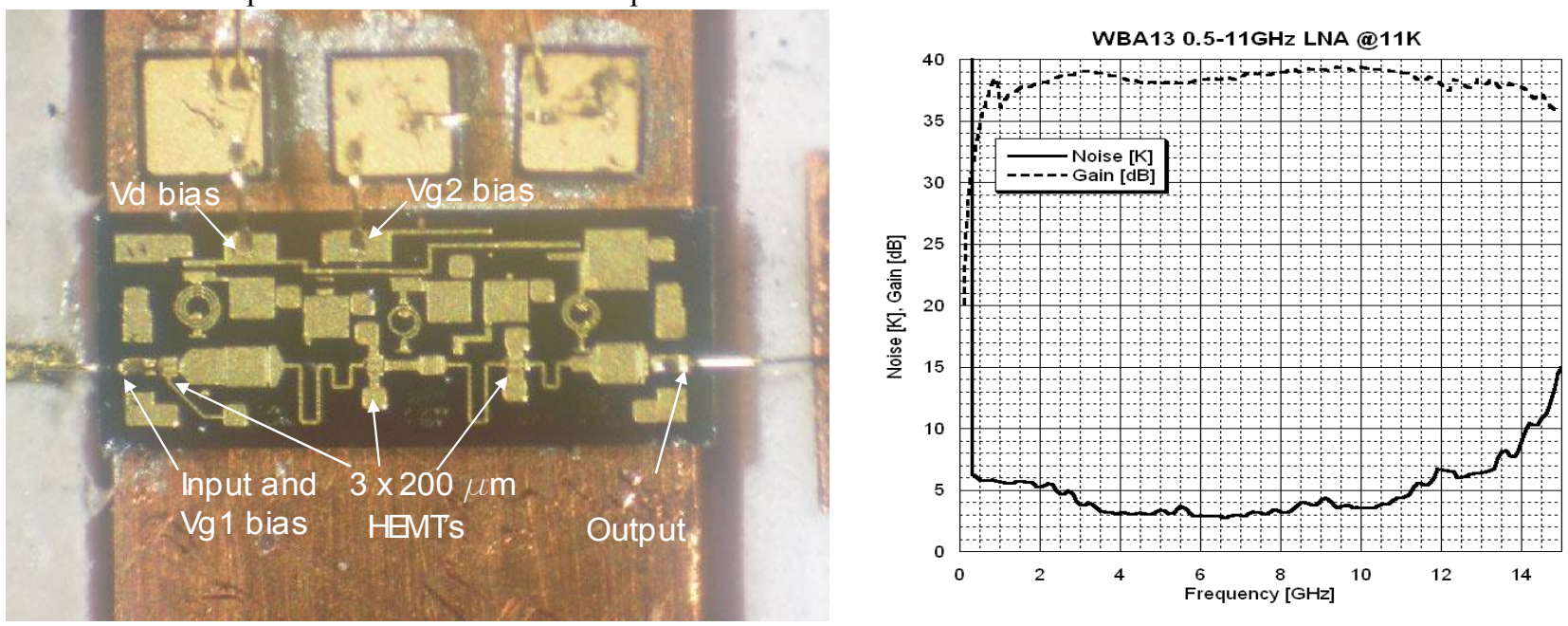

Figure 3 (left) Close-up view of a wideband MMIC amplifier chip (WBA13). The WBA12 and WBA13 are similar circuits with either two or three InP HEMT stages producing $\sim 25 \mathrm{~dB}$ and $\sim 35 \mathrm{~dB}$ of gain, respectively. (right) Noise temperature and gain of WBA13. WBA12 has same noise versus frequency characteristic but $\sim 10 \mathrm{~dB}$ less gain.

\section{Wide IF band 1 MM Receivers for CARMA}

All receivers were tested in a BIMA cryostat, where the LO is injected with a 0.3 mil Mylar beam splitter into the dewar input lens. The $\mathrm{LO}+\mathrm{RF}$ signal is matched to the input waveguide of the mixer with a scalar feedhorn which is bolted to the $4 \mathrm{~K}$ stage. Y-factor tests were highly automated. Tuning of the LO, biasing of the mixer, and operation of the chopper wheel were all under computer control. LO power was monitored by measuring the current through the voltage biased mixer. For a cold load we used a thin-walled Styrofoam box containing RF absorbing foam immersed in liquid nitrogen. We did not attempt to correct our noise temperature calculations for optics losses, and we assumed hot and cold load temperatures were $295 \mathrm{~K}$ and $77 \mathrm{~K}$, respectively. The receiver IF power was amplified outside the cryostat by a $1-10$ GHz JCA 110-317 with $30 \mathrm{~dB}$ gain. Broadband IF power was measured through a DC $-6 \mathrm{GHz}$ lowpass filter with an HP 8484 power sensor attached to an HP 436 power meter. Power levels were computer monitored. Measurements for hot or cold loads were made by setting the LO frequency and power, stepping the mixer bias voltage, and recording the IF power. Best noise temperatures are usually obtained by voltage biasing the SIS junction near the center of the first photon step in the I-V curve. Over the RF band of this device the optimal bias voltage varies from $1.9-2.5 \mathrm{mV}$. Y factor measurements for each frequency were made at optimum LO power level.

\subsection{Receiver I: SIS mixer followed by WBA13 MMIC coaxial amplifier module}

We tried the simplest approach first to increase mixer IF bandwidth, by attaching a current design BIMA mixer module with SMA connector output to a coaxial module containing a WBA13 MMIC through a 1" section of 0.085 stainless steel semirigid coaxial cable. The cable thermally isolates the $4 \mathrm{~K}$ mixer and $10 \mathrm{~K}$ MMIC. The devices were attached by heat 
straps to separate stages of the BIMA cryostat, which is cooled by a three stage Gifford-McMahon refrigerator ${ }^{8,9}$. This arrangement minimizes the heat load from the biased MMIC on the SIS mixer.

Figure 4 shows Receiver I, including an open view of the mixer module and the assembled module attached to the MMIC amplifier module. The mixer chip sits in a suspended waveguide channel between the poles of a fixed magnet. The mixer chip IF pad is bonded with a 1 mil gold wire to a $50 \Omega$ microstrip. A bias tee is shown with series $10 \mathrm{~K}$ current limiting resistors on the bias lines and a $100 \Omega$ resistor shunting the junction impedance for stable voltage biasing.

The best double-sideband (DSB) receiver temperature $\mathrm{T}_{\text {rec,DSB }}$ of Receiver I as a function of LO frequency is shown in Figure 5. The results show improvement over the results for a BIMA mixer followed by an L band amplifier (Figure 2). From $205-240 \mathrm{GHz} \mathrm{T}_{\mathrm{rec}, \mathrm{DSB}}$ is $7-10 \mathrm{~K}$ lower. At the high frequency end of the band, $\mathrm{T}_{\mathrm{rec}, \mathrm{DSB}}$ is about the same.
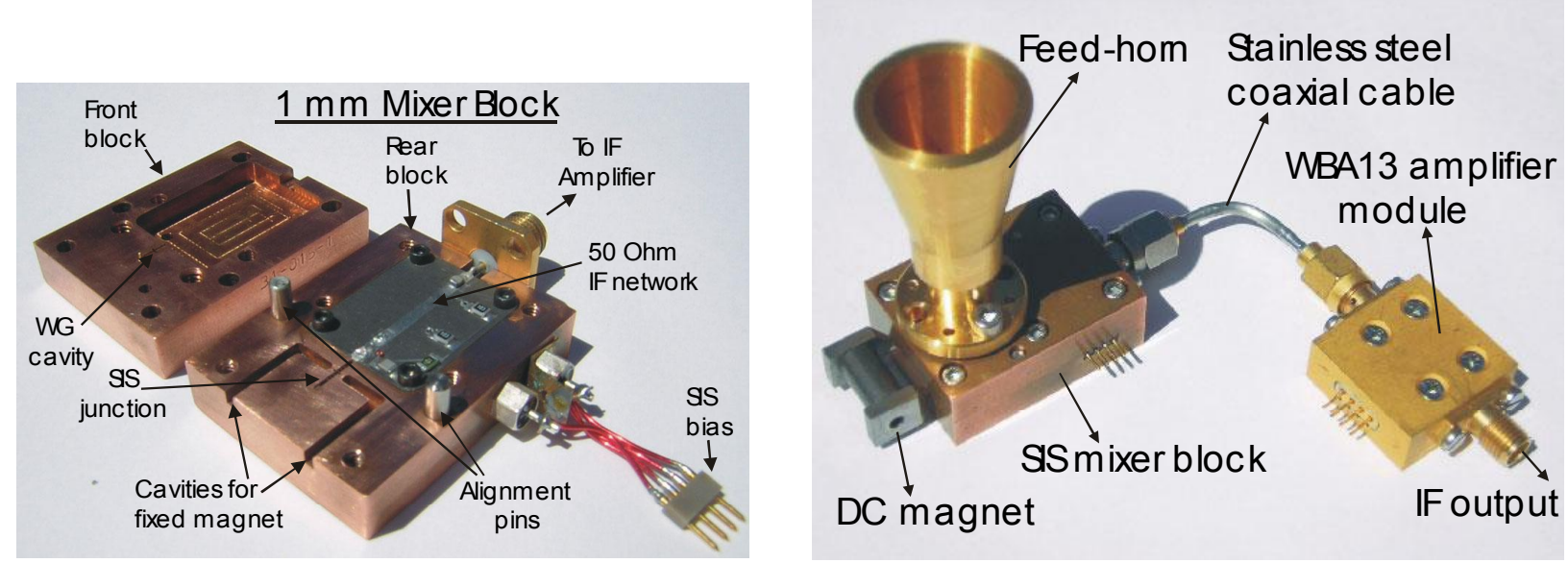

Figure 4 Open view of the BIMA SIS mixer module (left) and view of Receiver I consisting of BIMA mixer module attached to WBA13 amplifier module through 1" section of stainless steel coax.

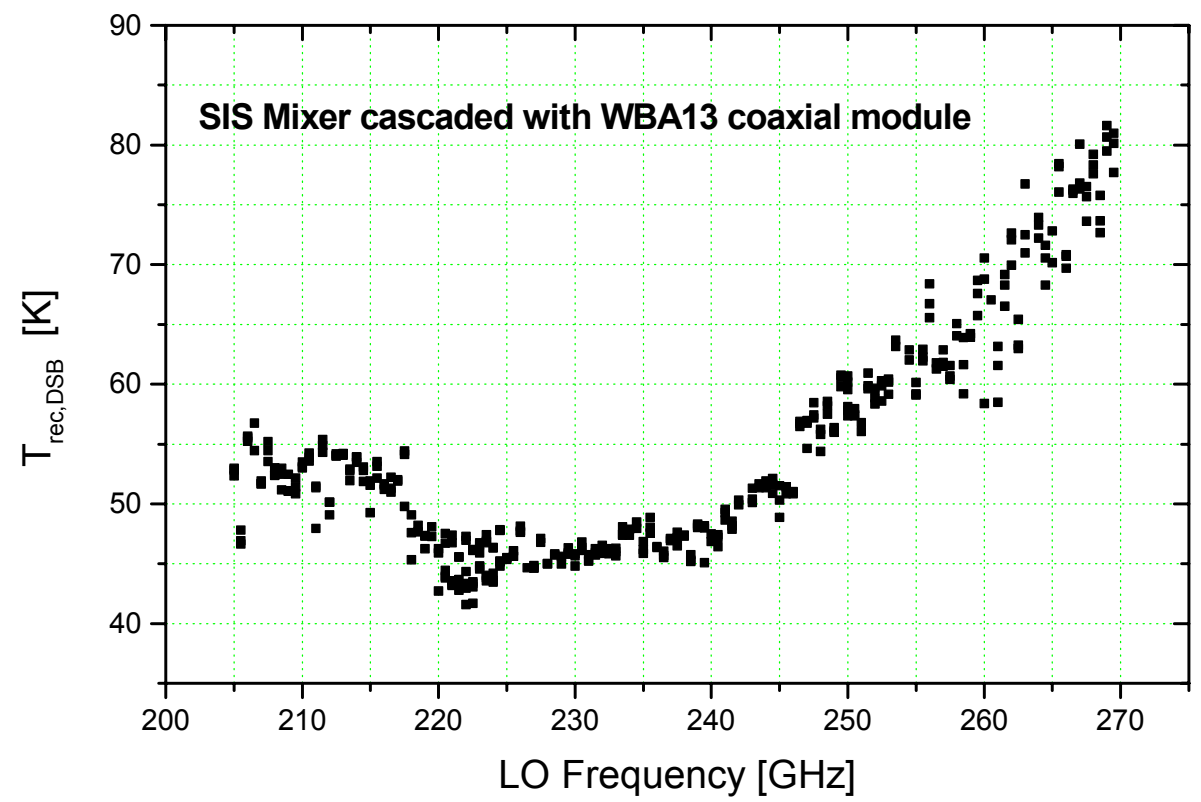

Figure 5 Receiver temperature versus LO frequency for Receiver I. The IF bandwidth was 0-6 GHz. 
Discrepancies between Receiver I and the BIMA receiver noise temperatures may be due to loss in a longer IF coaxial cable connecting the SIS mixer to the L Band LNA in the BIMA receiver.

We measured the gain and noise temperature of Receiver I across the IF passband by feeding the output of the JCA amplifier into an Agilent E4407B spectrum analyzer. The resolution bandwidth was set to $1 \mathrm{MHz}$, and power was integrated over $25 \mathrm{MHz}$. Figure 6 shows output power as a function of IF frequency for hot and cold terminations when the $\mathrm{LO}$ frequency is tuned to $225 \mathrm{GHz}$. The power has a 5 -- $10 \mathrm{dBm}$ ripple over $1 \mathrm{GHz}$ intervals.

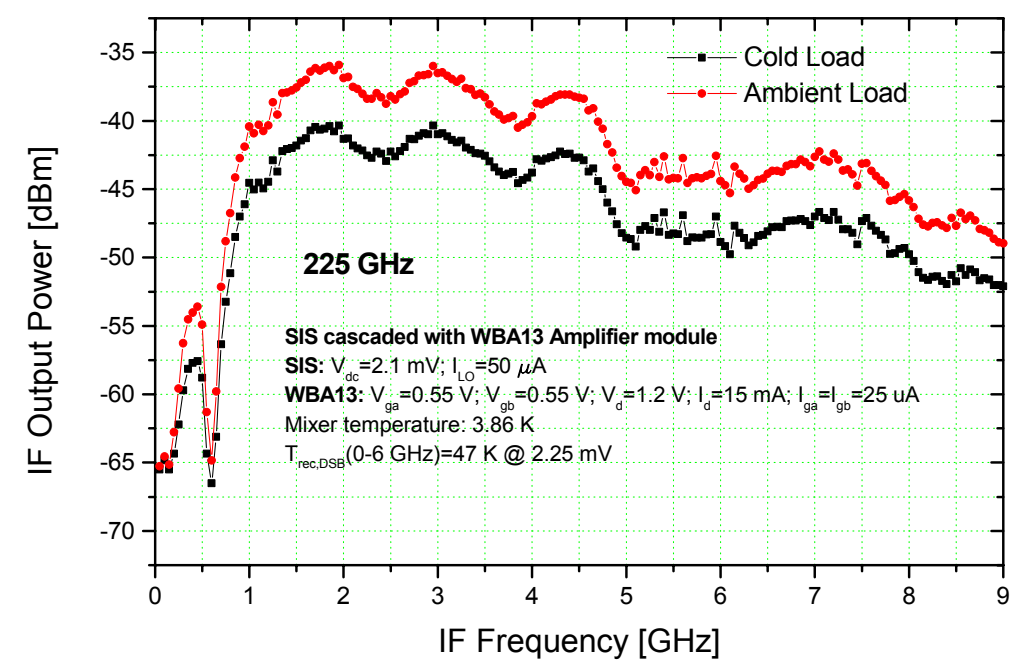

Figure 6 IF output power versus IF frequency for Receiver I at LO frequency of $225 \mathrm{GHz}$. Large ripples observed are due to impedance mismatch between the mixer and MMIC modules. These ripples become more severe for LO frequencies near the edges of the mixer RF band.

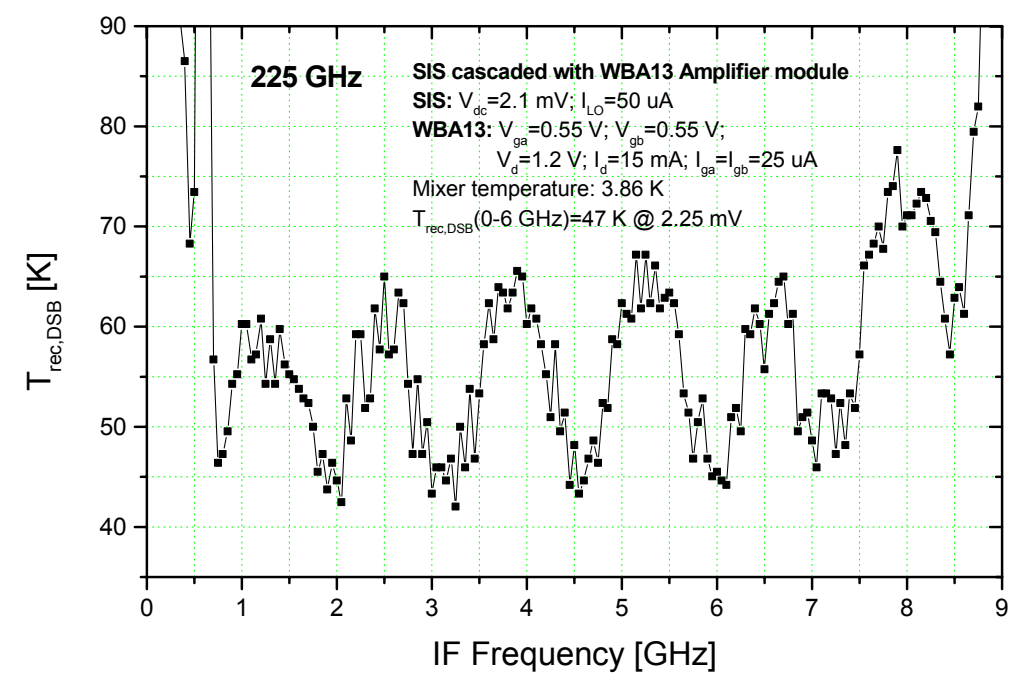

Figure 7 Receiver temperature of Receiver I as a function of IF frequency at LO frequency of $225 \mathrm{GHz}$. Large ripples are due to an impedance mismatch between mixer and MMIC modules. 
$\mathrm{T}_{\text {rec,DSB }}$ derived from these curves, shown in Figure 7, rises sharply below $0.7 \mathrm{GHz}$ and above $8.5 \mathrm{GHz}$, denoting the edges of the senstivity band. Clearly, the BIMA mixers can be modified to have at least a $4 \mathrm{GHz}$ IF bandwidth. The $20 \mathrm{~K}$ ripples in receiver temperature are due to a standing wave on the transmission lines linking the SIS junction to the WBA13, indicating significant impedance mismatch. Even more severe ripples occur for $v_{\mathrm{LO}}<215 \mathrm{GHz}$ and $v_{\mathrm{LO}}>245$ GHz. Such large gain and sensitivity variations in the IF passband would be difficult to calibrate out so either the mixer needs to be followed by a wideband isolator, which would require us to increase the IF band to 4-8 GHz, necessitating changes to the OVRO mixers and the downconverter for the correlator; or a more sophisticated matching network is required; or mixer and MMIC need to be electrically closer, which would increase the frequency interval of the standing wave and make passband calibration easier. Receiver II, described below, is our first successful attempt at closely integrating mixer and MMIC.

\subsection{Receiver II: SIS mixer integrated with WBA12}

We directly integrated a WBA12 amplifier ( $\sim 25 \mathrm{~dB}$ gain) into a modified BIMA mixer block. Figure 8 shows an open view of the SIS/MMIC module and a closeup view of the circuitry linking the SIS mixer and InP MMIC amplifier. Integration
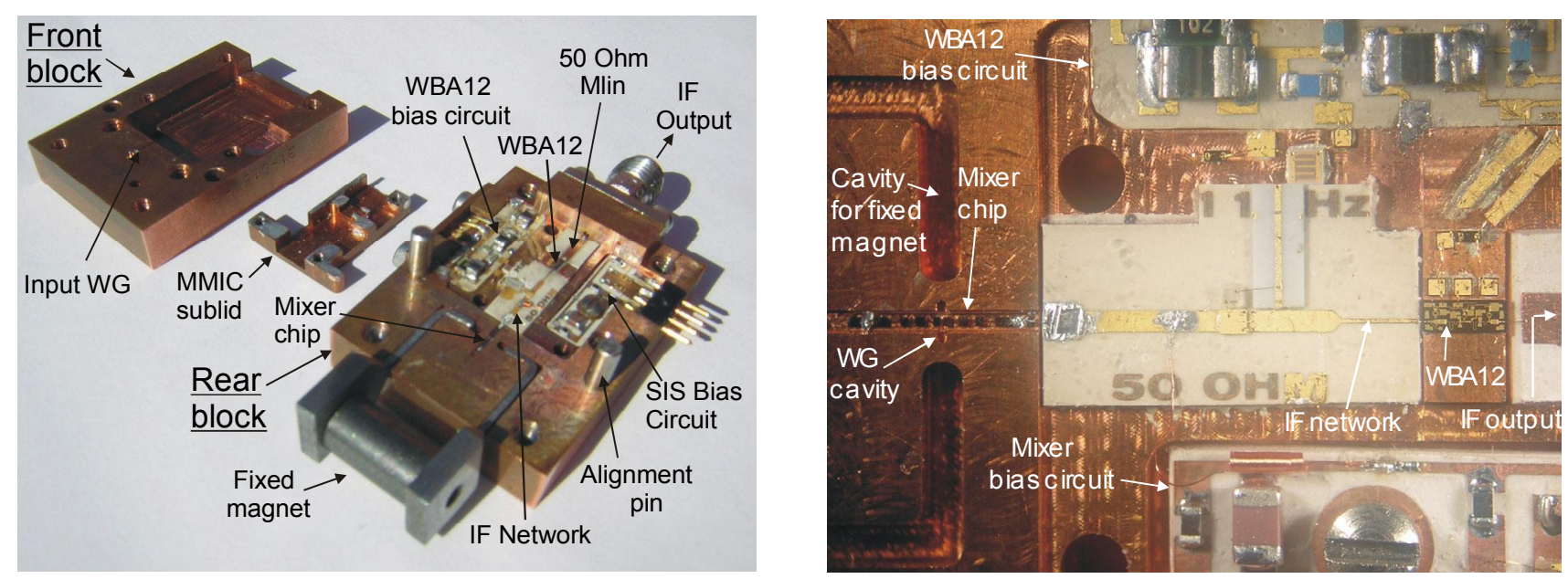

Figure 8 Open view of Rcceiver II showing SIS junction integrated with WBA 12 amplifier chip(left). Matching network shown in detail (right) presents $50 \Omega$ to junction and $100 \Omega$ to MMIC input gate.

required installing a bias circuit for the MMIC, which supplies two independent gate voltages and a shared drain current for the three HEMT stages. Dielectric Labs bias network chips (p/n B28BHBFNO1) filter radio frequency interference from the SIS bias lines; lumped element low pass filters protect the DC lines on the MMIC bias board. The DC bias lines for the SIS junction and MMIC input gate are attached to $50 \Omega$ transmission lines coupled by a Dielectric Labs $6.8 \mathrm{pF}$ capacitor. The complete matching circuit presents $50 \Omega$ impedance to the mixer chip IF port, and the MMIC gate capacitance is tuned by a thin (inductive) microstrip line. An irregularly shaped sublid, attached to the module body with 0-80 screws, encloses the matching circuit and MMIC in a rectangular waveguide with cutoff frequency above the maximum operating frequency of the amplifier. This prevents the MMIC output signal from coupling back to the input. Feedback can lead to out of band oscillations which will degrade the gain stability and noise temperature of the receiver. We found that perfect contact of the sides of the sublid near the MMIC chip was essential for stable operation so we applied a thin layer of indium solder around the sublid edges to guarantee excellent RF continuity. 


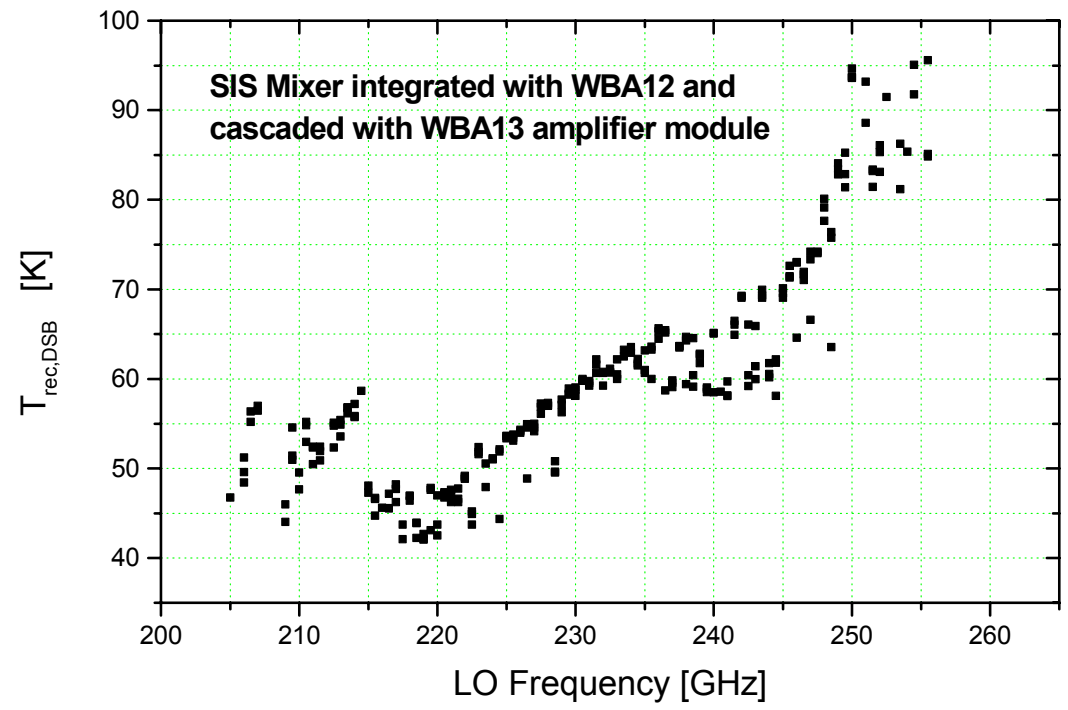

Figure 9 Receiver temperature of Receiver II as a function of LO frequency.. The sharply rising receiver temperatures for LO frequency $>230 \mathrm{GHz}$ possibly due to SIS probe misalignment in suspended stripline channel.

We originally assembled Receiver II with a WBA13 ( $\sim 35 \mathrm{~dB}$ gain) but found that we could not prevent the MMIC from oscillating at $4 \mathrm{~K}$. The reduced gain of the WBA12, combined with a modification of the matching circuit, allowed us to stably bias the MMIC when cold. But the decreased gain made it necessary to follow with a wideband amplifier on the 10 $\mathrm{K}$ stage - the coaxial WBA13 module - in order to achieve the desired receiver noise temperatures. These are shown plotted as a function of LO frequency in Figure 9.

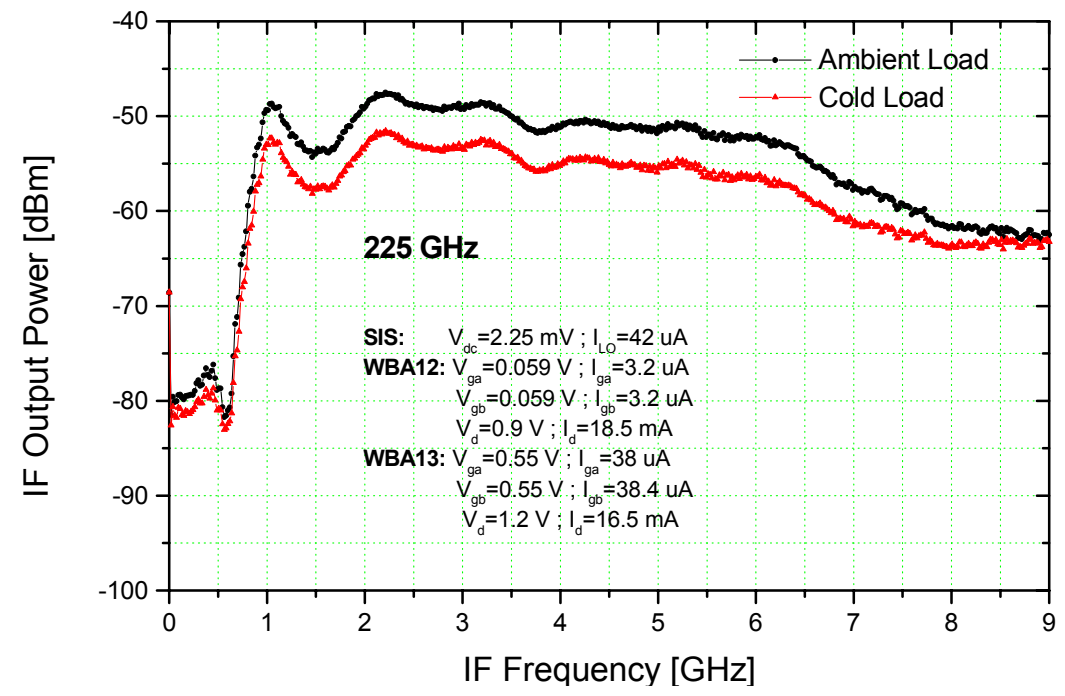

Figure 10 IF output power of Receiver II for LO frequency of $225 \mathrm{GHz}$. Ripples above $2 \mathrm{GHz}$ significantly smaller than measured for Receiver I

Receiver II performs well from $210-230 \mathrm{GHz}$, achieving noise temperatures as low as $43 \mathrm{~K}$, but $\mathrm{T}_{\text {rec,DSB }}$ rises steeply above $240 \mathrm{GHz}$. Since the SIS chip is identical for Receivers I and II it seems plausible that the reduced RF bandwidth is due either to the misalignment of the chip in the suspended substrate channel, thereby reducing the bandwidth of the waveguide probe or to the matching circuit between mixer and MMIC. Figure 10, however shows that the IF power of Receiver II rolls off more smoothly with frequency than that of Receiver I. The effect of standing waves on sensitivity variation versus IF frequency has been reduced. However, two standing waves are now apparent - one with a period of 6 $\mathrm{GHz}$ and an amplitude of $15 \mathrm{~K}$, and a second with the same period as that observed in the Receiver I IF band (1 GHz), but 
with $5-10 \mathrm{~K}$ amplitude. Overall, these gain variations should be easy to calibrate out at a spectrometer. The $6 \mathrm{GHz}$ standing wave is likely due to mismatch between SIS mixer and the WBA12 while the second faster period variation is probably due to a standing wave between the WBA12 and WBA13. It should also be noted that the sensitivity bandwidth of receiver II is $6 \mathrm{GHz}$ while that of Receiver I is $8 \mathrm{GHz}$. Also, the widenband noise temperature measurement for 225 $\mathrm{GHz}$ is $5-10 \mathrm{~K}$ lower than the average of the narrowband results. This may be due to offsets in the spectrum analyzer.

A minor problem is the $20 \mathrm{~mW}$ power dissipated by the WBA12 which raises the SIS operating temperature $\sim 0.7 \mathrm{~K}$. This is likely to raise $\mathrm{T}_{\text {rec,DSB }}$ less than $5 \mathrm{~K}$. (see Figure 3).

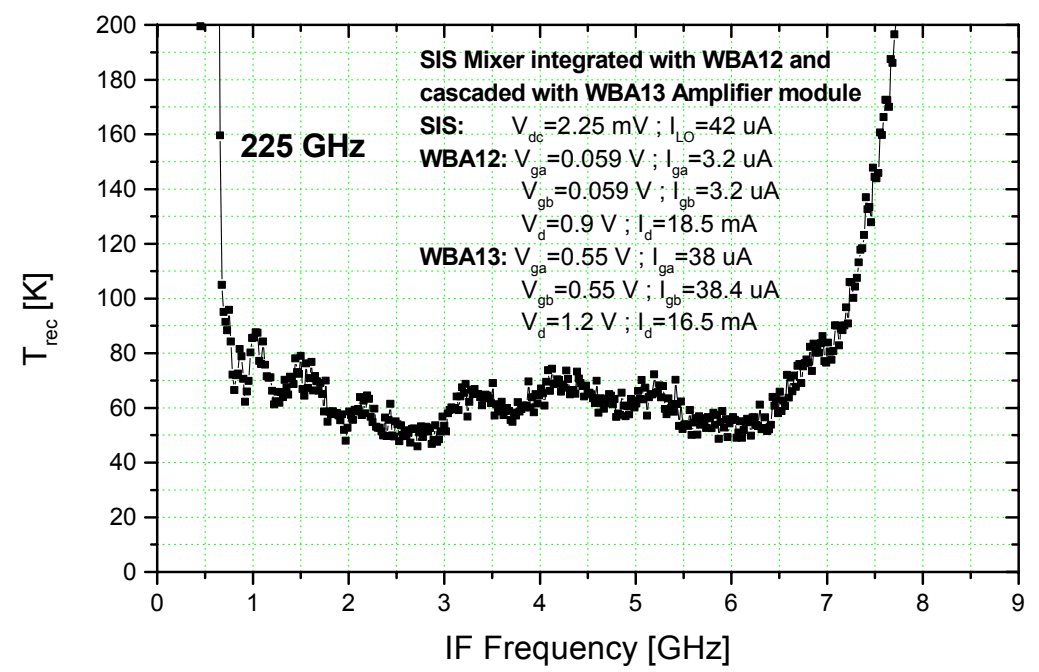

Figure 11 Receiver II narrow band noise temperature measured as a function IF frequency.

\subsection{Receiver III: Thermally split integrated SIS/WBA13 module}

Our second attempt to integrate SIS device and MMIC, shown in Figure 12, involved radically altering the mixer module so that it has thermally independent stages for SIS and MMIC. A TeCu mixer block at $4 \mathrm{~K}$ supported by fiberglass tabs
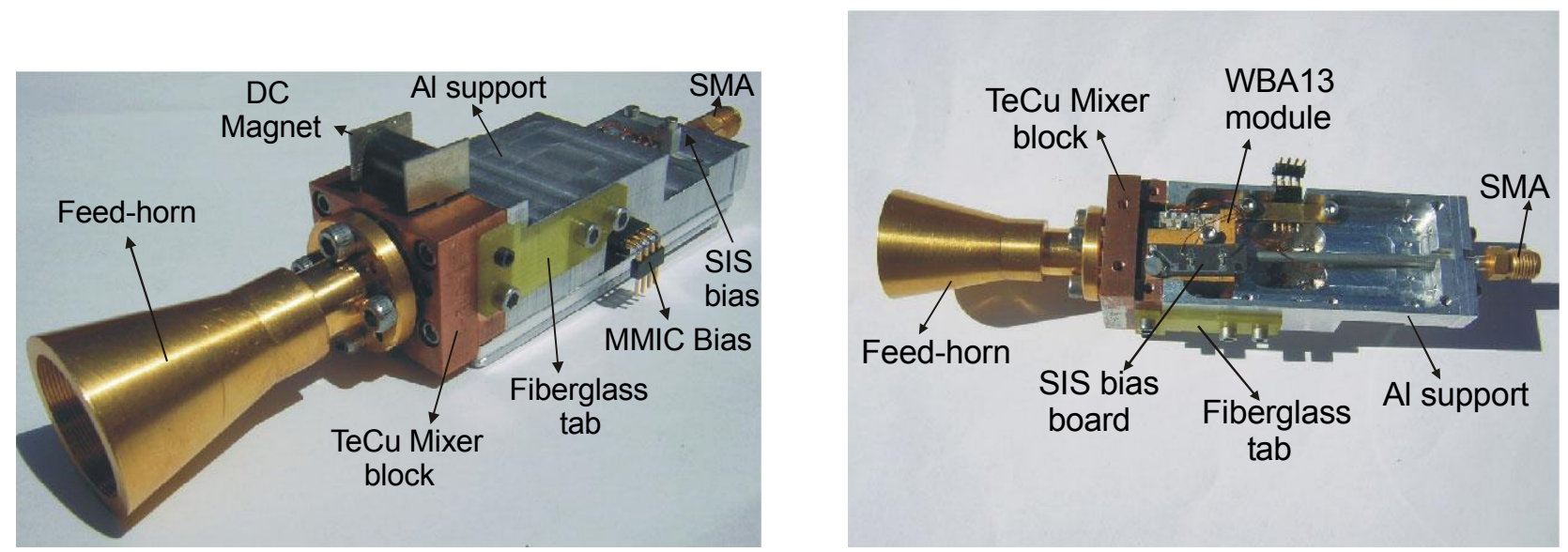

Figure 12 Receiver III assembled view (left) and open view (right). The TeCu mixer block at $4 \mathrm{~K}$ is thermally isolated from the WBA13 submodule at $10 \mathrm{~K}$ by fiberglass standoff tabs. A pair of $0.5 \times 200 \mathrm{mil}$ Au wires attach the mixer IF port to the $100 \Omega$ input microstrip of the amplifier module. 
stands off 10 mil from an aluminum support block maintained at $10 \mathrm{~K}$. From our experience, keeping the WBA13 chip at $10 \mathrm{~K}$ makes it easier to stably bias. And by thermally isolating the SIS junction we can maintain it at the lowest possible temperature for optimum sensitivity. The WBA13 MMIC is embedded in a submodule with bias circuitry; this submodule was designed by Wadefalk and Weinreb for the Allen Telescope Array front end. We were motivated to use the submodule since it represents a significant amount of outsourced preassembly and testing of wideband receiver components. The submodule is mounted in the support block with 2-56 screws. Two four-pin Microtech connectors supply bias to mixer and MMIC. The SIS bias board is attached to the top to the submodule and the DC bias attachment is made through a 10 turn inductor to the microstrip input of the MMIC submodule. The DC/IF port of the SIS mixer is connected by two $0.5 \times 200$ mil Au wires to the input of the MMIC module - the ground wire is soldered just below the open end of the suspended substrate SIS mixer channel and attaches to a point on the MMIC submodule just beneath the input microstrip.

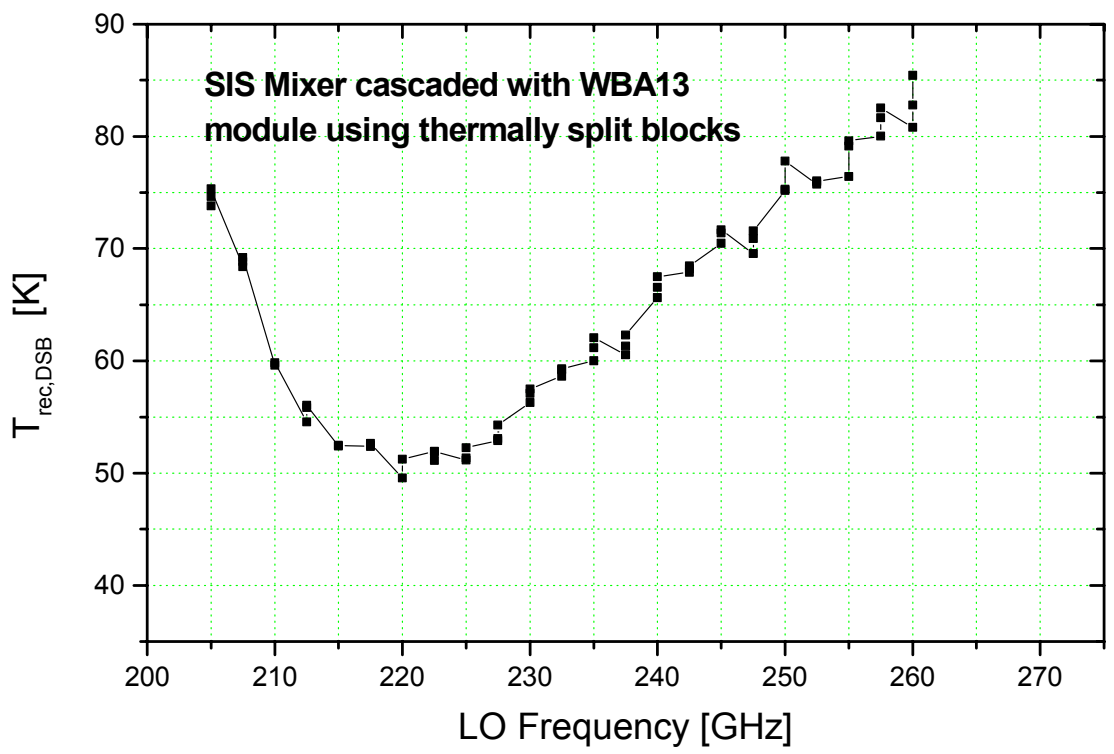

Figure 13 Integrated DC -6 GHz receiver temperature measued for Receiver III for LO frequency range $205-260 \mathrm{GHz}$. Receiver temps. appear somewhat higher than Receiver I because the IF noise temperature rises above $4 \mathrm{GHz}$

Test results of Receiver III are encouraging. Figure 13 shows that the receiver temperature versus frequency is similar to that of Receiver I. The somewhat higher noise temperatures measured for Receiver III could be due to absorbed water vapor in the Styrofoam cold load dewar. Particularly striking is the smoothness of the receiver IF power curves shown in Figure 14. This is likely the combined result of close proximity of mixer and MMIC in addition to a better matching circuit in the WBA13 amplifier submodule, which presents a $100 \Omega$ real impedance to the mixer. $T_{\text {rec,DSB }}$ as a function of $v_{\mathrm{IF}}$, shown in Figure 15, is nearly flat for $0.5-4.5 \mathrm{GHz}$. Extension of the IF band to higher frequencies might be achieved by reducing the length of the signal and ground wires connecting mixer and MMIC. This would cause a slight increase in the operating temperature of the SIS mixer but in the current arrangement heat loading from the MMIC raises the mixer temperture $0.1 \mathrm{~K}$. For example, modelling shows that reducing the lead lengths from 200 mil to 20 mil could increase the IF bandwidth of Receiver III to that of Receiver I $(8 \mathrm{GHz})$ while raising the integrated receiver temperature less than $5 \mathrm{~K}$. 


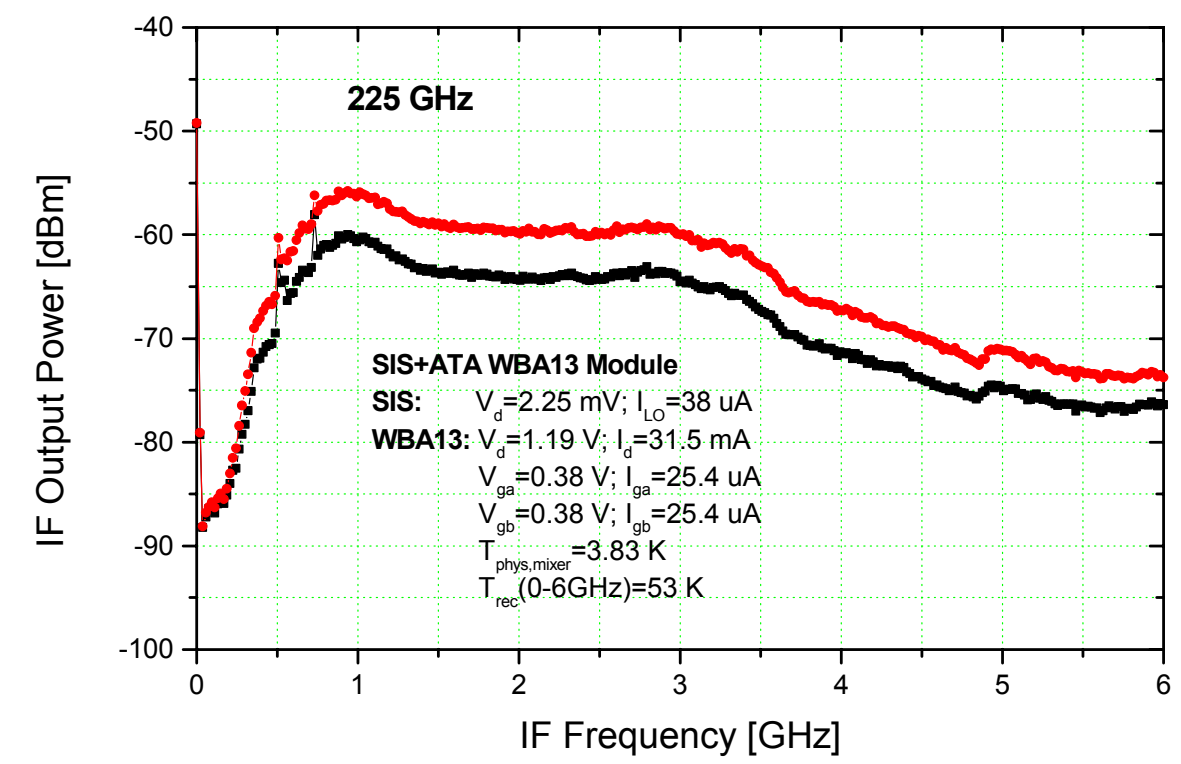

Figure 14 IF output power versus frequency for Receiver III.

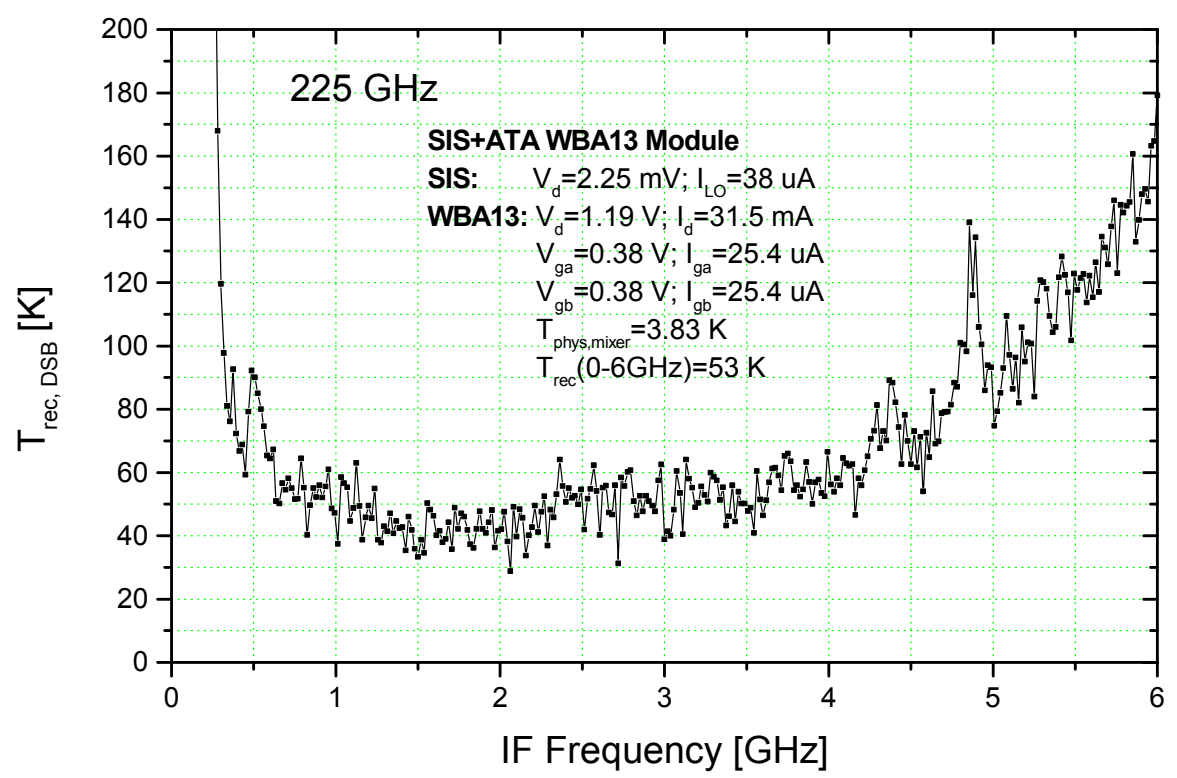

Figure 15 Receiver III noise temperatures measured as a function of IF frequency at a LO frequency of $225 \mathrm{GHz}$. Sensitivity level is remarkably constant for $0.5-4 \mathrm{GHz}$. This receiver has adequate sensitivity for CARMA and is compatible with the CARMA correlator.

\section{CONCLUSION}

We attempted three schemes to broaden the IF bandwidth of the BIMA $1 \mathrm{~mm}$ receiver to match or exceed the $4 \mathrm{GHz}$ bandwidth of the CARMA correlator, resulting in three receivers. For each receiver, noise temperature measurements for $205-270 \mathrm{GHz}$ were made by measuring Y factors corresponding to IF power integrated from DC to $6 \mathrm{GHz}$. Also, 
variations in power and sensitivity across the IF band were measured at $225 \mathrm{GHz}$. Receiver I was made by linking the BIMA SIS mixer module at $4 \mathrm{~K}$ to a $0.5-11 \mathrm{GHz}$ MMIC amplifier module at $10 \mathrm{~K}$ (WBA13) with a 1 " section of $50 \Omega$ stainless steel coaxial cable. While the receiver temperatures across the RF band were satisfactory and the IF bandwidth was nearly $8 \mathrm{GHz}$, we measured ripple in receiver IF power arising from an impedance mismatch between mixer and MMIC that would be difficult to calibrate out.

In Receiver II we were able to decrease the frequency of this ripple by directly integrating a MMIC amplifier into a modified BIMA $1 \mathrm{~mm}$ mixer module. However, we had problems with stably biasing a WBA13 at $4 \mathrm{~K}$ so we substituted a lower gain MMIC chip, the WBA12. This chip produces only $25 \mathrm{~dB}$ gain so a separate WBA13 amplifier stage at $10 \mathrm{~K}$ had to be cascaded with the SIS/WBA12 module. This resulted in an IF power curve with two IF standing waves : one between mixer and WBA12; the other between the WBA12 and the WBA13. The broadband noise temperatures across the RF band looked satisfactory for $210-240 \mathrm{GHz}$, but a misalignment of the mixer chip may be the cause of the narrower measured overall RF bandwidth. The $20 \mathrm{~mW}$ of DC power supplied to the WBA12 raised the SIS physical temperature only $\sim 0.7 \mathrm{~K}$ but this is unlikely to have degraded $\mathrm{T}_{\text {rec,DSB }}$ appreciably.

Receiver III, based on a completely redesigned mixer module, maintains thermal isolation between the $4 \mathrm{~K}$ SIS junction and the $10 \mathrm{~K}$ WBA13 amplifier. Two $0.5 \times 200$ mil Au wires link the IF port of the SIS mixer to the $100 \Omega$ microstrip input of a WBA13 submodule embedded in the thermally split receiver module. Heat dissipation in the amplifier increased the physical temperature of the SIS junction less than $0.1 \mathrm{~K}$. $\mathrm{T}_{\mathrm{rec}, \mathrm{DSB}}$ was satisfactory for LO frequencies of 205 $-260 \mathrm{GHz}$. Gain and sensitivity vary acceptably across the IF bandwidth of $0.5-4.5 \mathrm{GHz}$. Reducing the length of the Au leads connecting mixer and MMIC from 200 mil to 20 mil should increase the IF bandwidth to as much as $8 \mathrm{GHz}$ without appreciably raising $T_{\text {rec,DSB }}$ across the band.

\section{REFERENCES}

1. D.P. Woody., "CARMA: A new heterogeneous millimeter-array interferometer", this conf., Proceedings of SPIE Vol. 5498

2. Padin, S., Woody, D.P., Stern, J.A., LeDuc, H.G., Blundell, R., Tong, C.-Y.E., and Pospieszalski, M., “An Integrated SIS

Mixer and HEMT IF Amplifier,” IEEE Trans. Microwave Theory Tech., vol. MTT-44, no. 6, pp. 987-990, June 1996

3. Engargiola, G., Plambeck, R. L., Wideband $3 \mathrm{~mm}$ SIS mixers operated with partial saturation, SPIE Proceedings Vol. 3357, pp 508-518, 1998 (Available also asbima.astro.umd.edu/memo/memo65.ps).

4. Blundell, R., Tong, C. E., Papa, D. C., Leombruno, R., Zhang, X., Paine, S., Stern, J. A., LeDuc, H. G., “A wideband fixed-tuned SIS receiver for $200 \mathrm{GHz}$ operation,” IEEE Trans. Microwave Theory Tech., 43, pp. 933-937, 1995.

5. Kerr, A.R., Pan, S, -K., Lichtenberger, A.W., and Huang, H.H.,"A tunerless SIS mixer for 200-280 GHz with low output

capacitance and inductance," Proceedings of the Ninth International Symposium of Space Terahertz Technology, “ pp. 195-203, 17 March 1998

6. Lauria E.F., Kerr A.R., Pospieszalski, M., Pan, S, -K., Effland, J.E., and Lichtenberger, A.W., “ 200-300 GHz SIS Mixer-Preamplifier with 8 GHz IF Bandwidth”, ALMA Memo 378, 7 June 2001

7. D. DeBoer.et al, "The Allen Telescope Array",this conf., Proceedings of SPIE Vol 5489

8. Plambeck, R.L., Thatte, N. Sykes, P. (1992) “A 4 K Gifford-McMahon Refrigerator for Radio Astronomy,” Proc. Int. Cryocooler Conf., Santa Fe, NM, Nov. 1992

9. Plambeck, R.L. 1998, "Long Term Performance of 4 K Gifford-McMahon Referigerators on the BIMA Array," in Advances in Cryogenic Engineering, vol 43 (P. Kittel, ed), New York: Plenum, pp. 1815-1821. 